

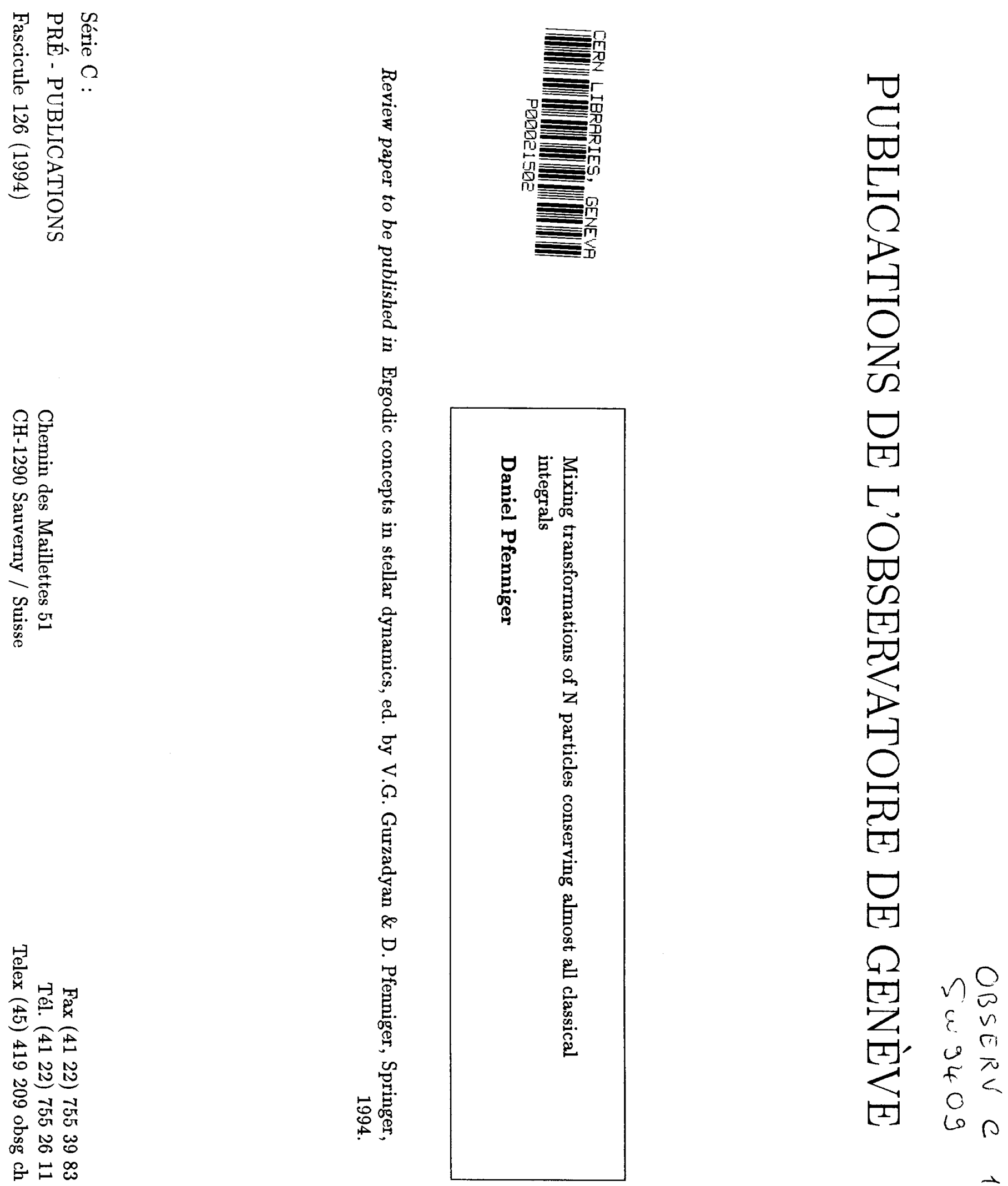


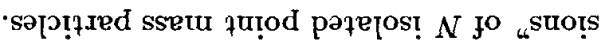

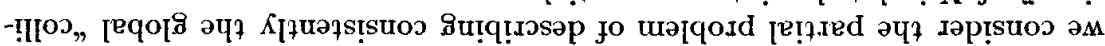

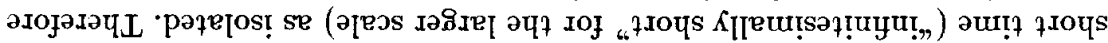

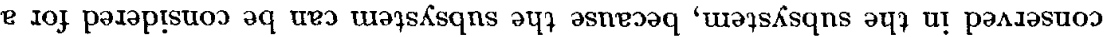

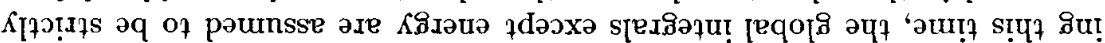

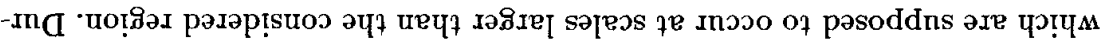

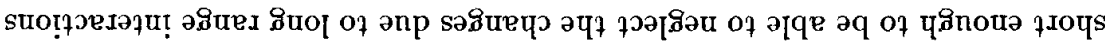

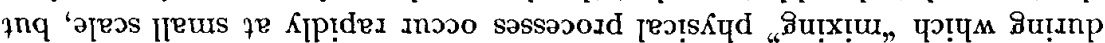

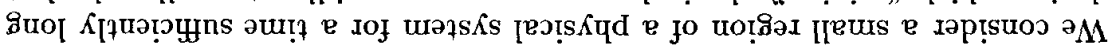

\section{smet uọłenxəsuo}

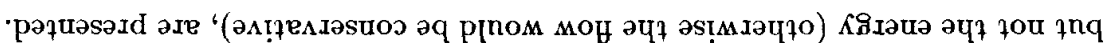

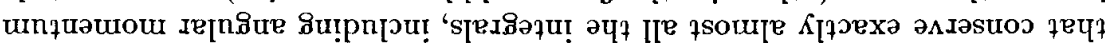

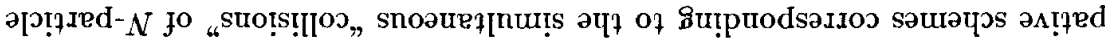
-тsstp ұse e ио stiǫฺ?

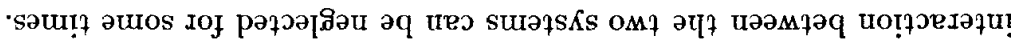

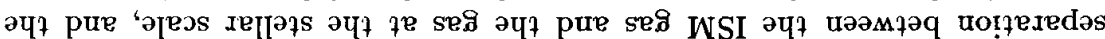

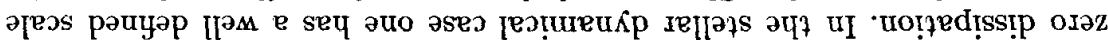

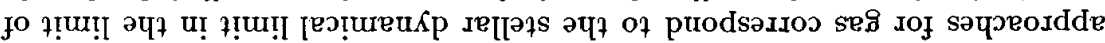

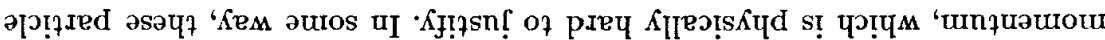

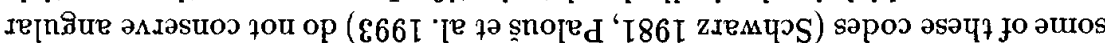

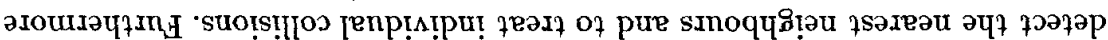

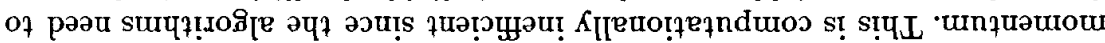

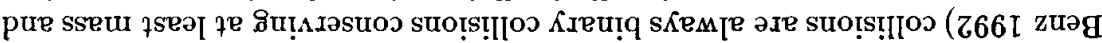

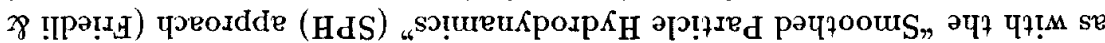

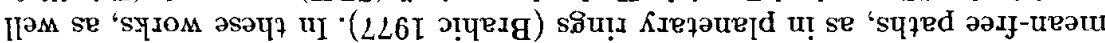

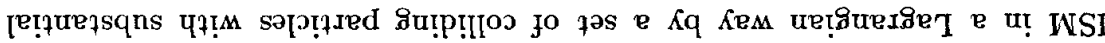

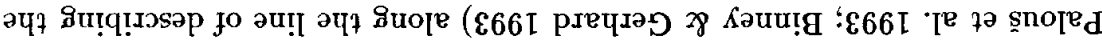

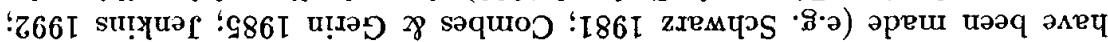

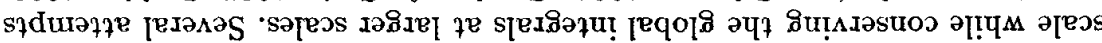

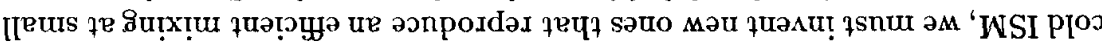

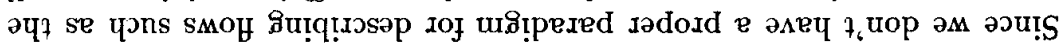

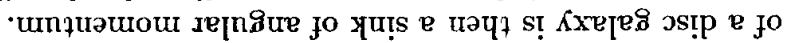

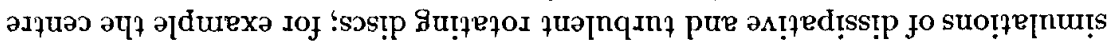

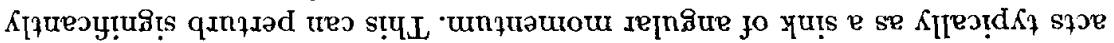

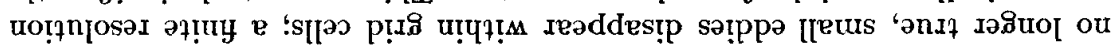

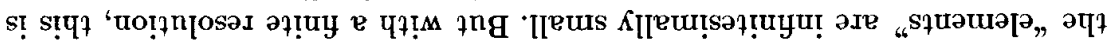

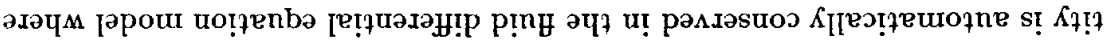

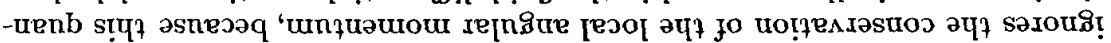




\title{
Mixing Transformations of N Particles Conserving Almost All Classical Integrals
}

\author{
Daniel PFENNIGER \\ Geneva Observatory, University of Geneva, CH-1290 Sauverny, Switzerland
}

\section{Paradigm for Inhomogeneous Mixing Flows}

The Navier-Stokes equation is often used as paradigm for modelling the gas in galaxies. Yet the interstellar medium (ISM) is far from being a smooth common flow. In fact the cold phase of the ISM, containing most of the gas mass, is essentially an inhomogeneous medium better described with a fractal model, and where the density can vary by 10 decades over distances much shorter than < 1 pc (Pfenniger \& Combes 1994). Typically the "mean-free path" of clumps at the $10-50$ pc scale, such as molecular clouds, is much larger than their size, and an hydrodynamical description with particles of the size of molecular clouds can be, and has been, tried. However molecular clouds collide and dissolve because they contain internal degrees of freedom due to the smaller clumps moving inside them. For a fractal gas there is no strong reason to choose a particular scale. The resolution required to follow such an inhomogeneous flow at the $100 \mathrm{pc}$ scale or larger makes the hydrodynamical approach hardly tractable with todays computers.

So for any practical smallest scale one can choose (not to be confused with a very small scale that might be retained in the future with very powerful computers), the differentiability of the flow required by the Navier-Stokes equation for modelling the cold ISM cannot be granted. Although the differential fluid equations are in principle inapplicable, those have nevertheless the main virtue to conserve locally some of the classical integrals at the smallest accessible scale. If the physical flow is highly turbulent and fractal at small scale it is illusory to believe that the simulated flow reproduces faithfully the detail of the physical flow. But if the physical flow mixes rapidly a part of its microscopic properties, as in statistical mechanics only the global integrals are conserved at larger scale and at least these quantities are relevant in simulations.

However the finite element Eulerian approach for describing fluids, either by grid schemes (van Albada 1985; Mulder \& Liem 1986; Athanassoula 1991) or by beam schemes (e.g. Sanders \& Prendergast 1974; van Albada et al. 1982) 


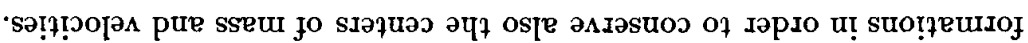

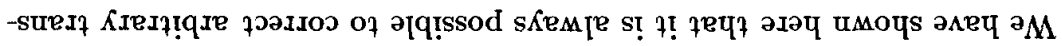

$$
\cdot{ }^{\prime} \boldsymbol{\Lambda}-\boldsymbol{\Lambda}=\mathbf{a} \text { pue }{ }^{\prime} \mathbf{X}-\mathbf{X}=\mathbf{0}
$$

шәчм $\Lambda={ }_{11} \Lambda$ рше $\mathbf{X}={ }_{\mu \prime} \mathbf{X}$ әмеч әм snчL

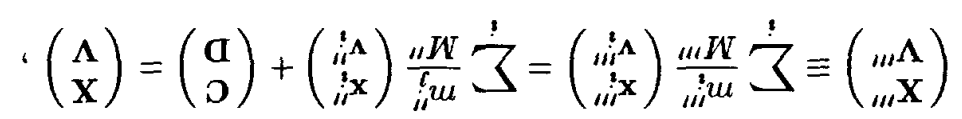

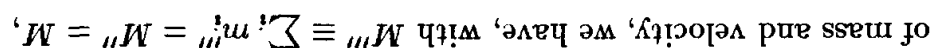

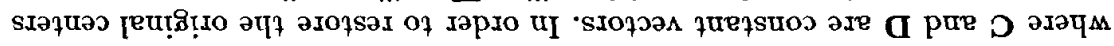

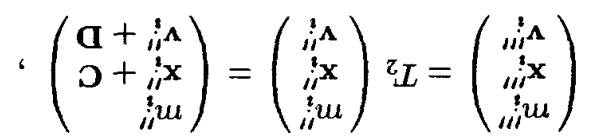

$: z^{2} L$ uotqeunojsuesł queqsuos e jo

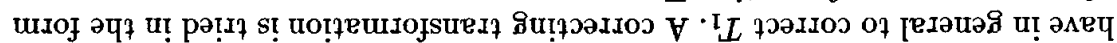

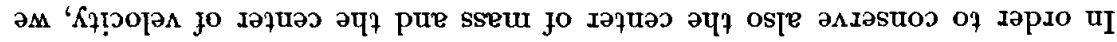

\section{иот̨влxаsuo}

'səsseur әл!ฺ!

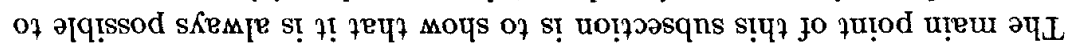

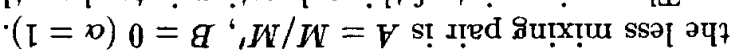

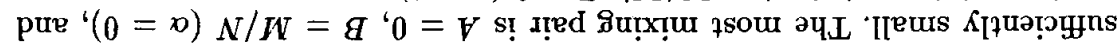

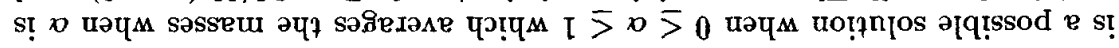

$$
\begin{aligned}
& \text { ' } \frac{N}{W}(x-\mathrm{I})=g \quad \text { ' } \frac{, W}{W} x=V \\
& \text { ป!̣ed aप7 ә[durexa IOH }
\end{aligned}
$$

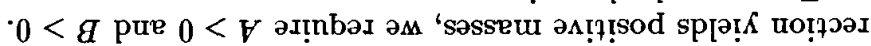
-

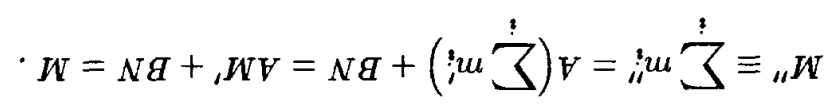

кjstipes plnoys

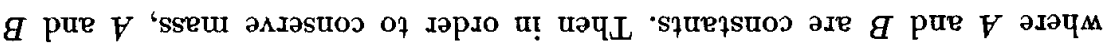

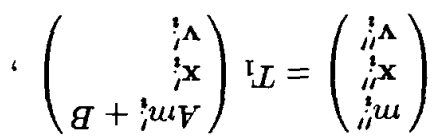

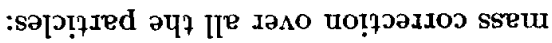

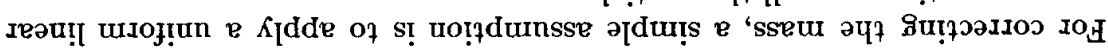

$$
\begin{aligned}
& \text { uompaxasuo sseW } \boldsymbol{\varepsilon} \cdot \boldsymbol{z}
\end{aligned}
$$

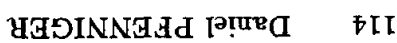




\subsection{Conservation Laws}

The $N$ particles are described in an inertial frame by their positive mass $m_{i}$, their positions $\mathbf{x}_{i}$, and their velocities $\mathbf{v}_{i}, i=1 \ldots N$. We require that the considered dissipative process conserves the total mass $M$, center of velocity $\mathbf{V}$ and center of mass $\mathbf{X}$

$$
M \equiv \sum_{i} m_{i}, \quad M \mathrm{~V} \equiv \sum_{i} m_{i} \mathbf{v}_{i}, \quad M \mathbf{X} \equiv\left(\sum_{i} m_{i} \mathbf{x}_{i}\right)-M \mathbf{V} t
$$

and the total angular momentum about its center of mass,

$$
\mathrm{L} \equiv \sum_{i} m_{i}\left(\mathbf{x}_{i}-\mathbf{X}\right) \wedge \mathbf{v}_{i}
$$

i.e. the integrals not involving a particular form of interaction. On the other hand the total energy is not supposed to be conserved. This is motivated by the fact that in many physical cases energy is the less well conserved quantity owing to the numerous possibilities of exchanging rapidly energy by radiative processes. In contrast, momentum and angular momentum can practically only be dissipated by mass exchanges (see, e.g., Shu 1992).

\subsection{Arbitrary Transformations of Coordinates}

First suppose that we apply an arbitrary transformation $T_{0}$ to the particles coordinates at a fixed time $t$. We also suppose that the particle masses can be transformed, but the new masses must remain positive. Since the time is not used, we can set $t=0$ in the following. This yields new masses $\left(m_{i}^{\prime}>0\right)$, positions $\left(\mathbf{x}_{i}^{\prime}\right)$, and velocities $\left(\mathbf{v}_{i}^{\prime}\right)$

$$
\left(\begin{array}{c}
m_{i}^{\prime} \\
\mathbf{x}_{i}^{\prime} \\
\mathbf{v}_{i}^{\prime}
\end{array}\right)=T_{0}\left(\begin{array}{c}
m_{i} \\
\mathbf{x}_{i} \\
\mathbf{v}_{i}
\end{array}\right) .
$$

In general, $T_{0}$ does not conserve the global integrals, yielding new integrals $M^{\prime}, \mathbf{X}^{\prime}, \mathbf{V}^{\prime}$ and $\mathbf{L}^{\prime}$. We suppose that the actual physical transformation is mixing, that is, except for the global invariants, the memory of the precise initial conditions are lost. So we should "correct" $T_{0}$ in order to restore a process that conserves the global integrals, except the energy. The corrections should be as uniform as possible for all particles, and should not use the detailed information about the old coordinates (the mixing process forgets the precise values of the old coordinates, but not the global integrals except the energy). 


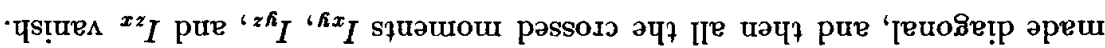

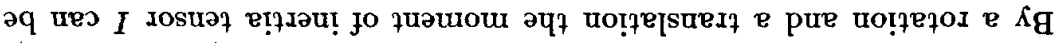

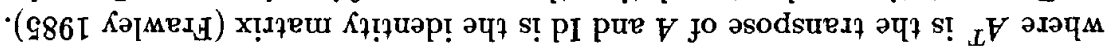

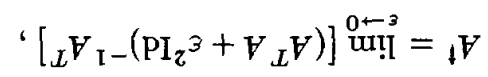

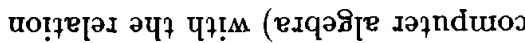

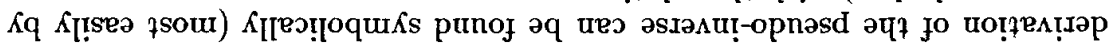

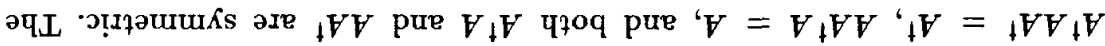

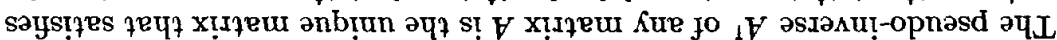

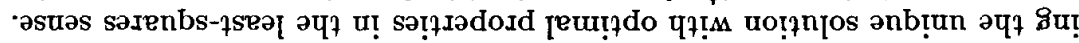

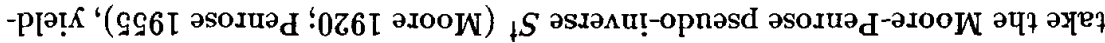

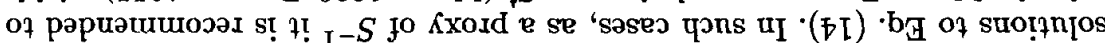

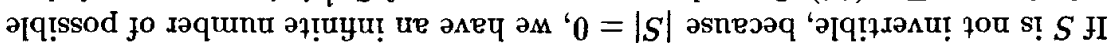

\section{sәsв}

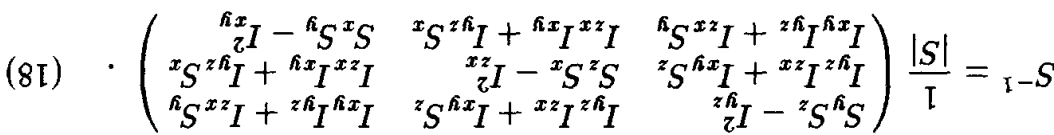

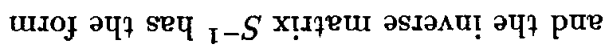

$$
\begin{aligned}
& { }^{\text {‘ }}{ }_{z}^{x} I{ }^{z} S-{ }_{z}^{x z} I^{\hbar} S-{ }_{z}^{z \hbar} I^{x} S-{ }^{x z} I^{z \hbar} I^{\hbar x} I z-{ }^{z} S^{\hbar} S{ }^{x} S=|S|
\end{aligned}
$$

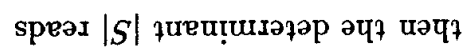

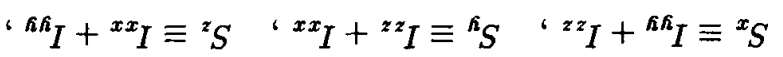

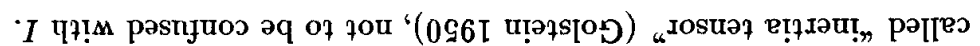

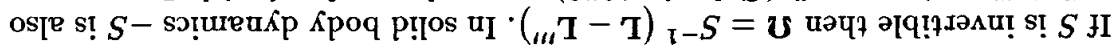

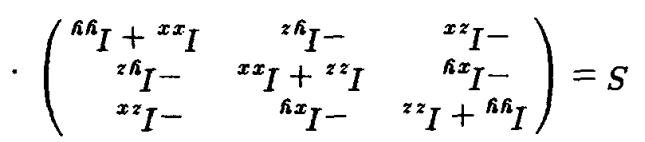

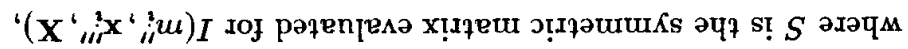

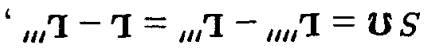

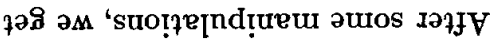

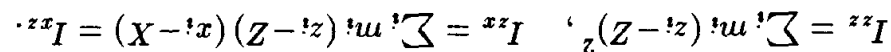

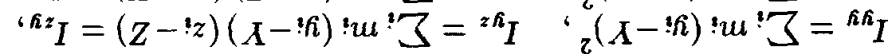

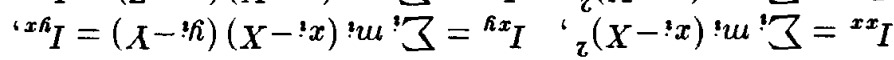




\subsection{Angular Momentum Conservation}

The transformations $T_{1}$ and $T_{2}$ provide a new total angular momentum $\mathrm{L}^{\prime \prime \prime}$ that is generally different from the original one $\mathbf{L}$. Since constant corrections are insufficient to conserve angular momentum, we adopt the next simplest hypothesis; we suppose a transformation of the velocities only, and having the same form for all particles except for a linear dependence on the own particle position with respect to the center of mass. Although it could be possible to set up more complicated correcting transformations involving both the positions and the velocities, we don't correct the positions on the ground of simplicity. In general, position transformations produce also potential energy changes, and forces modify velocities to first order, and positions to second order.

In order to modify the total angular momentum without changing the center of velocity, the simplest velocity correction must be orthogonal to and linear with the radius vector to the center of mass. Therefore we require

$$
\left(\begin{array}{c}
m_{i}^{\prime \prime \prime} \\
\mathbf{x}_{i}^{\prime \prime \prime} \\
\mathbf{v}_{i}^{\prime \prime \prime}
\end{array}\right)=T_{3}\left(\begin{array}{l}
m_{i}^{\prime \prime \prime} \\
\mathbf{x}_{i}^{\prime \prime \prime} \\
\mathbf{v}_{i}^{\prime \prime \prime}
\end{array}\right)=\left(\begin{array}{l}
m_{i}^{\prime \prime \prime} \\
\mathbf{x}_{i}^{\prime \prime \prime} \\
\mathbf{v}_{i}^{\prime \prime \prime}+\mathbf{\Omega} \wedge\left(\mathbf{x}_{i}^{\prime \prime \prime}-\mathbf{X}\right)
\end{array}\right),
$$

where $\boldsymbol{\Omega}$ is a constant vector to specify. This transformation is a solid rotation in velocity space that obviously conserves the mass and the center of mass, but it conserves also the center of velocity, since

$$
\begin{aligned}
M^{\prime \prime \prime \prime} \mathbf{V}^{\prime \prime \prime \prime} & \equiv \sum_{i} m_{i}^{\prime \prime \prime} \mathbf{v}_{i}^{\prime \prime \prime}=\sum_{i} m_{i}^{\prime \prime \prime} \mathbf{v}_{i}^{\prime \prime \prime}+\Omega \wedge \underbrace{\left(\sum_{i} m_{i}^{\prime \prime \prime} \mathbf{x}_{i}^{\prime \prime \prime}-M \mathbf{X}\right)}_{=0 \text { by Eqs. (8) and (9) }} \\
& =\sum_{i} m_{i}^{\prime \prime \prime} \mathbf{v}_{i}^{\prime \prime \prime}=M \mathrm{~V} .
\end{aligned}
$$

By using the identity $\mathbf{a} \wedge(\mathbf{b} \wedge \mathbf{a})=\mathbf{a}^{2} \mathbf{b}-(\mathbf{a} \cdot \mathbf{b}) \mathbf{a}$, then the new angular momentum is

$$
\begin{aligned}
\mathbf{L}^{\prime \prime \prime \prime} & \equiv \sum_{i} m_{i}^{\prime \prime \prime \prime}\left(\mathbf{x}_{i}^{\prime \prime \prime \prime}-\mathbf{X}\right) \wedge \mathbf{v}_{i}^{\prime \prime \prime \prime} \\
& =\sum_{i} m_{i}^{\prime \prime \prime}\left(\mathbf{x}_{i}^{\prime \prime \prime}-\mathbf{X}\right) \wedge \mathbf{v}_{i}^{\prime \prime \prime}+\sum_{i} m_{i}^{\prime \prime \prime}\left(\mathbf{x}_{i}^{\prime \prime \prime}-\mathbf{X}\right) \wedge\left[\mathbf{\Omega} \wedge\left(\mathbf{x}_{i}^{\prime \prime \prime}-\mathbf{X}\right)\right] \\
& =\mathbf{L}^{\prime \prime \prime}+\sum_{i} m_{i}^{\prime \prime \prime}\left(\mathbf{x}_{i}^{\prime \prime \prime}-\mathbf{X}\right)^{2} \mathbf{\Omega}-\sum_{i} m_{i}^{\prime \prime \prime}\left[\left(\mathbf{x}_{i}^{\prime \prime \prime}-\mathbf{X}\right) \cdot \mathbf{\Omega}\right]\left(\mathbf{x}_{i}^{\prime \prime \prime}-\mathbf{X}\right)
\end{aligned}
$$

If we require that the new angular momentum $\mathbf{L}^{\prime \prime \prime \prime}$ equals the original one $\mathbf{L}$ we can solve this linear equation for $\boldsymbol{\Omega}$ in term of the coordinates $\mathbf{x}_{i}^{\prime \prime \prime}$ only. This is a familiar equation of solid body dynamics (see e.g. Goldstein 1950).

\subsubsection{Moment of Inertia Tensor}

First we define the moment of inertia tensor $I\left(m_{i}, \mathbf{x}_{i}, \mathbf{X}\right)$ about the center of mass $\mathbf{X}$, where $\mathbf{x}_{i}=\left(x_{i}, y_{i}, z_{i}\right), \mathbf{X}=(X, Y, Z)$, 


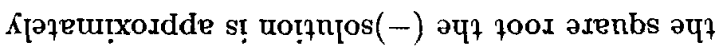

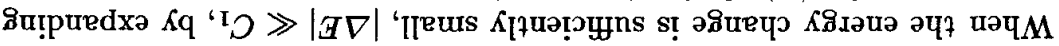

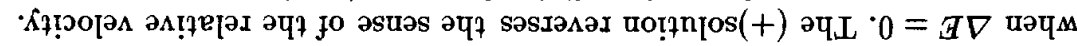

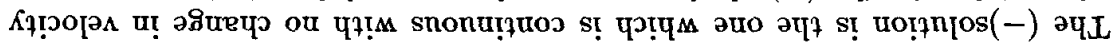

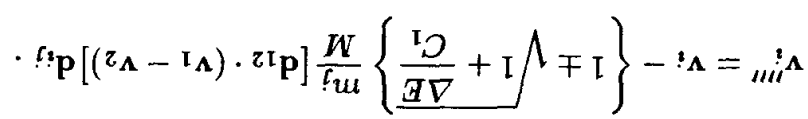

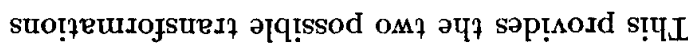

$$
\frac{\mathrm{I} D}{\underline{\underline{B}} \boldsymbol{\nabla}}+\mathrm{I} \Lambda \mp=0
$$

'puy pue 'o

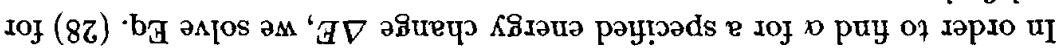

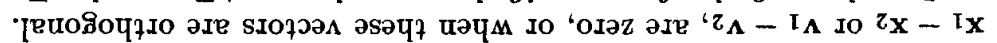

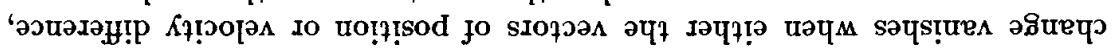

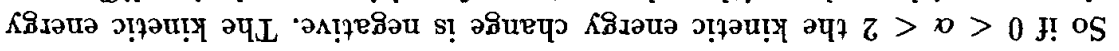

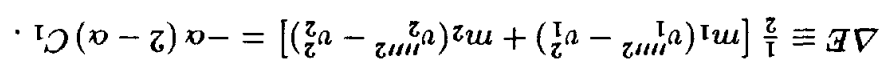

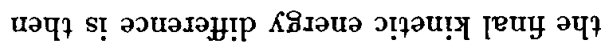

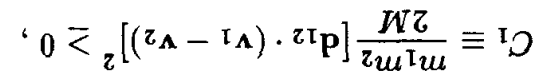

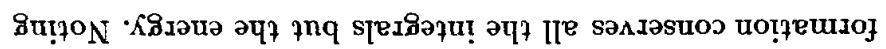

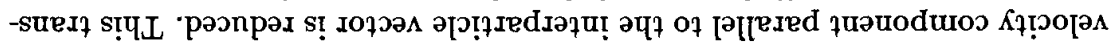

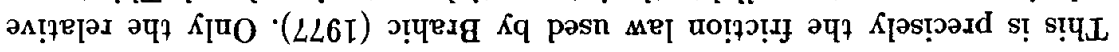

$$
\frac{\left|l_{\mathrm{x}}-?_{\mathrm{x}}\right|}{l_{\mathrm{x}}-?_{\mathrm{x}}}=? ! p
$$

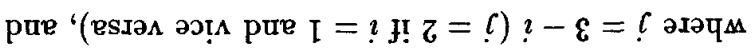

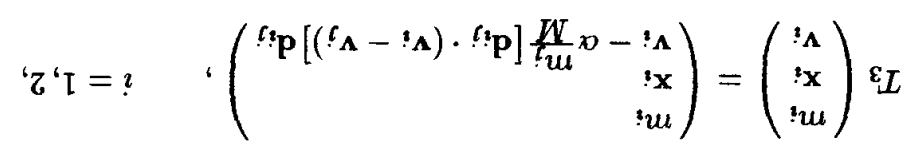

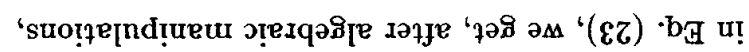

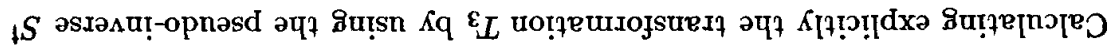

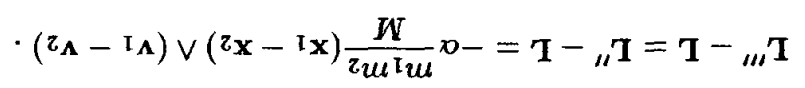

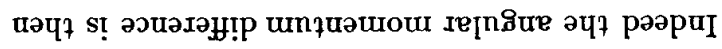

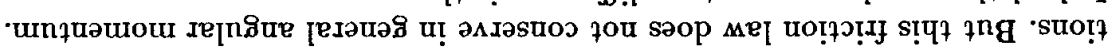

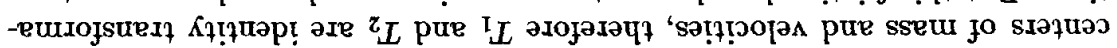

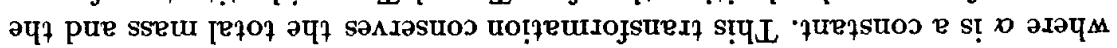


In that case $S$ is also diagonal and is not invertible only in the case where at least two of the three non-negative tensor components $I_{x x}, I_{y y}$ and $I_{z z}$ vanish (see Eq. (15)). This means that all the points are colinear, i.e. have the form $\mathbf{x}_{i}=\mathbf{a} t_{i}+\mathbf{b}$, where $\mathbf{a} \equiv\left\{a_{x}, a_{y}, a_{z}\right\}$ and $\mathbf{b}$ are constant vectors.

In this case, $S$ takes the form

$$
S=\frac{K}{|\mathbf{a}|^{2}}\left(\begin{array}{ccc}
a_{y}^{2}+a_{z}^{2} & -a_{x} a_{y} & -a_{z} a_{x} \\
-a_{x} a_{y} & a_{z}^{2}+a_{x}^{2} & -a_{y} a_{z} \\
-a_{z} a_{x} & -a_{y} a_{z} & a_{x}^{2}+a_{z}^{2}
\end{array}\right)
$$

where

$$
K \equiv I_{x x}+I_{y y}+I_{z z}=\frac{1}{M} \sum_{i} \sum_{j>i} m_{i} m_{j}\left(t_{i}-t_{j}\right)^{2} .
$$

If $K>0$ then the use of Eq. (19) leads to the pseudo-inverse $S^{\dagger}$

$$
S^{\dagger}=K^{-2} S \text {. }
$$

If $K=0$ then all the points are concomitant and $S^{\dagger}=0$.

\subsubsection{Case $N=2$}

In this particular case $S$ is always not invertible since two points are always colinear. Explicitly the pseudo-inverse $S^{\dagger}$ is

$$
\begin{aligned}
& S^{\dagger}=\frac{m_{1}+m_{2}}{m_{1} m_{2}} \frac{1}{\left(\mathbf{x}_{1}-\mathbf{x}_{2}\right)^{2}} \times \\
& \times\left(\begin{array}{ccc}
\left(y_{1}-y_{2}\right)^{2}+\left(z_{1}-z_{2}\right)^{2} & -\left(x_{1}-x_{2}\right)\left(y_{1}-y_{2}\right) & -\left(z_{1}-z_{2}\right)\left(x_{1}-x_{2}\right) \\
-\left(x_{1}-x_{2}\right)\left(y_{1}-y_{2}\right) & \left(z_{1}-z_{2}\right)^{2}+\left(x_{1}-x_{2}\right)^{2} & -\left(y_{1}-y_{2}\right)\left(z_{1}-z_{2}\right) \\
-\left(z_{1}-z_{2}\right)\left(x_{1}-x_{2}\right) & -\left(y_{1}-y_{2}\right)\left(z_{1}-z_{2}\right) & \left(x_{1}-x_{2}\right)^{2}+\left(y_{1}-y_{2}\right)^{2}
\end{array}\right) .
\end{aligned}
$$

Of course, if $\mathbf{x}_{1}=\mathbf{x}_{2}$, we have further degeneracy, and then $S^{\dagger}=0$.

\section{Examples: Linear Friction Laws}

\subsection{Binary Interactions}

Often dissipative particle schemes (Brahic 1977, Schwarz 1981) use binary interactions. In this case one can consider successively pairs of particles, so we need to consider a dissipative law for $N=2$. As a first guess a linear friction law with respect to the center of velocity is attempted

$$
T_{0}\left(\begin{array}{c}
m_{i} \\
\mathbf{x}_{i} \\
\mathbf{v}_{i}
\end{array}\right)=\left(\begin{array}{l}
m_{i} \\
\mathbf{x}_{i} \\
\mathbf{v}_{i}-\alpha\left(\mathbf{v}_{i}-\mathbf{V}\right)
\end{array}\right), \quad i=1,2
$$




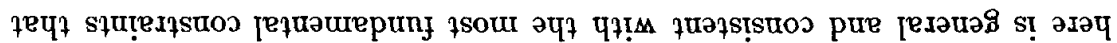

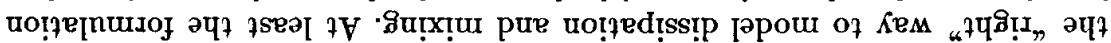

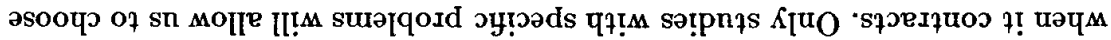

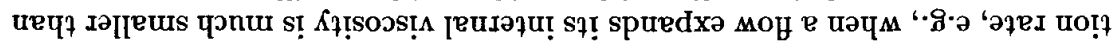

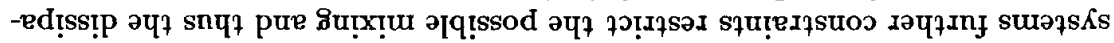

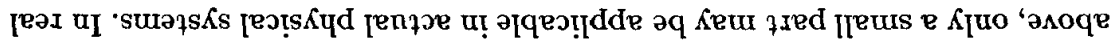

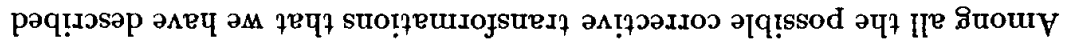

-onjes

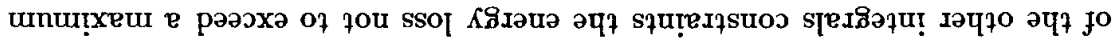

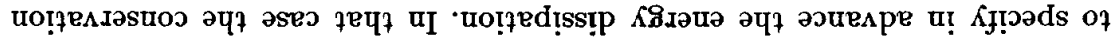

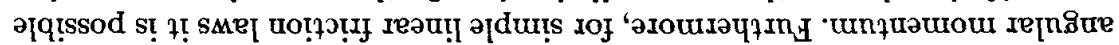

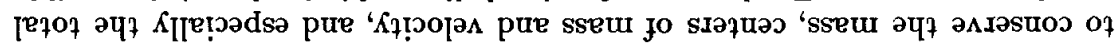

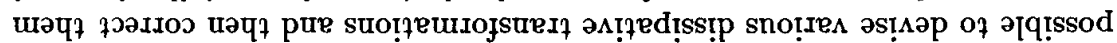

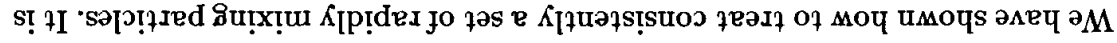

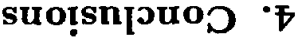

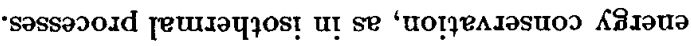

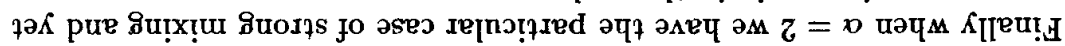

$$
\text { - } D / G \nabla \wedge-\approx 0
$$

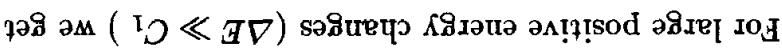

$$
\text { - } \mathrm{L} \rho / G \nabla \frac{\mathrm{z}}{\mathrm{L}}-\approx 0
$$

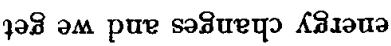

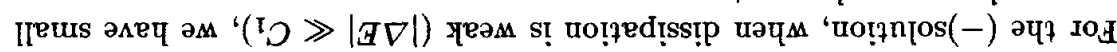

$$
\text { - }
$$

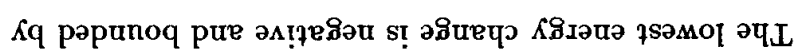

$\cdot(0 \leftarrow B \nabla$ иачм $0 \leftarrow x)$ uo!fed

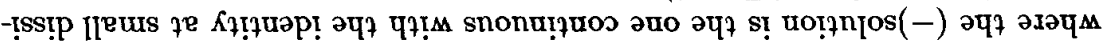

$$
\text { ' } \frac{\mathrm{I} D}{\underline{A} \nabla}+\mathrm{I} \Lambda \mp \mathrm{I}=0
$$

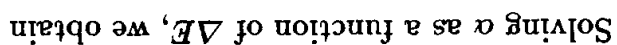

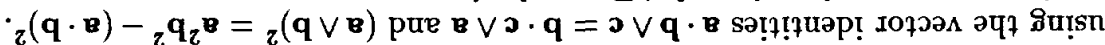

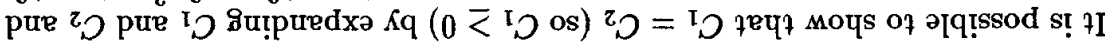

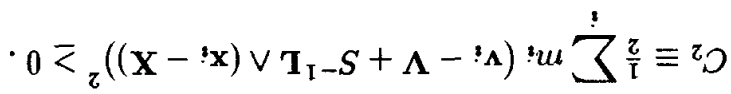

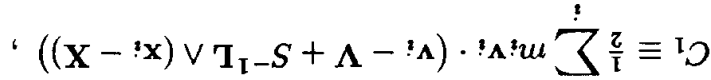




$$
\mathbf{v}_{i}^{\prime \prime \prime \prime} \approx \mathbf{v}_{i}+\frac{\Delta E}{m_{i} \mathbf{d}_{12} \cdot\left(\mathbf{v}_{1}-\mathbf{v}_{2}\right)} \mathbf{d}_{i j} .
$$

When the energy change is large and positive, $\Delta E \gg C_{1}$, the approximate solution reads

$$
\mathbf{v}_{i}^{\prime \prime \prime \prime} \approx \mathbf{v}_{i} \mp \sqrt{\frac{2 \Delta E}{M} \frac{m_{j}}{m_{i}}} \mathbf{d}_{i j} .
$$

The minimum energy change compatible with this type of dissipation is given by

$$
\Delta E \geq-C_{1} .
$$

\section{2 $N$ Interacting Particles}

Instead of considering particle interactions by pairs, it is much more efficient to group the interactions with larger $N$. This is justified if the mixing process has time to mix the properties of the $N$ particles during a finite time-step. If a linear friction law is used for an arbitrary number $N$ of particles, we would have

$$
\left(\begin{array}{c}
m_{i}^{\prime \prime} \\
\mathbf{x}_{i}^{\prime \prime} \\
\mathbf{v}_{i}^{\prime \prime}
\end{array}\right)=T_{0}\left(\begin{array}{l}
m_{i} \\
\mathbf{x}_{i} \\
\mathbf{v}_{i}
\end{array}\right)=\left(\begin{array}{l}
m_{i} \\
\mathbf{x}_{i} \\
\mathbf{v}_{i}-\alpha \mathbf{v}_{i}
\end{array}\right),
$$

where $\alpha$ is a constant. Then $T_{1}$ is an identity transformation and

$$
\left(\begin{array}{c}
m_{i}^{\prime \prime \prime} \\
\mathbf{x}_{i}^{\prime \prime \prime} \\
\mathbf{v}_{i}^{\prime \prime \prime}
\end{array}\right)=T_{2}\left(\begin{array}{l}
m_{i} \\
\mathbf{x}_{i} \\
\mathbf{v}_{i}
\end{array}\right)=\left(\begin{array}{l}
m_{i} \\
\mathbf{x}_{i} \\
\mathbf{v}_{i}-\alpha\left(\mathbf{v}_{i}-\mathbf{v}\right)
\end{array}\right) .
$$

The transformation $T_{3}$ is too complicated to give in closed form, but it is straightforward to evaluate numerically Eqs. (13), (15), and (18) efficiently with a computer, since the algorithm is proportional to $N$. Also in this linear case we do not need to evaluate $\mathbf{L}^{\prime \prime \prime}$ since one obtains,

$$
S \boldsymbol{\Omega}=\mathbf{L}-\mathbf{L}^{\prime \prime \prime}=\alpha \mathbf{L}, \quad \Longrightarrow \quad \mathbf{\Omega}=\alpha S^{-1} \mathbf{L},
$$

where $S^{-1}$ has to be replaced by $S^{\dagger}$ in case of degeneracy. Finally,

$$
\mathbf{v}_{i}^{\prime \prime \prime \prime}=\mathbf{v}_{i}-\alpha\left(\mathbf{v}_{i}-\mathbf{V}+S^{-1} \mathbf{L} \wedge\left(\mathbf{x}_{i}-\mathbf{X}\right)\right) .
$$

The kinetic energy difference $\Delta E$ is given by

$$
\Delta E=\frac{1}{2} \sum_{i} m_{i}\left(\mathbf{v}_{i}^{\prime \prime \prime \prime}{ }^{2}-\mathbf{v}_{i}^{2}\right)=-2 \alpha C_{1}+\alpha^{2} C_{2},
$$

where 


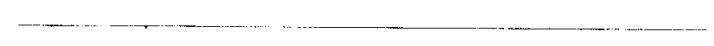

